\title{
MR Brain Real Images Segmentation Based Modalities Fusion and Estimation Et Maximization Approach
}

\author{
ASSAS Ouarda * \\ Department of Computer Science, \\ Laboratory Pure and Applied Mathematics (LPAM) \\ University of M'sila \\ M'sila, Algeria
}

\begin{abstract}
With the development of acquisition image techniques, more data coming from different sources of image become available. Multi-modality image fusion seeks to combine information from different images to obtain more inferences than can be derived from a single modality. The main aim of this work is to improve cerebral IRM real images segmentation by fusion of modalities (T1, T2 and DP) using estimation et maximizatio Approach (EM). The evaluation of adopted approaches was compared using four criteria which are: the standard deviation (STD), entropy of information (IE), the coefficient of correlation (CC) and the space frequency (SF). The experimental results on MRI brain real images prove that the adopted scenarios of fusion approaches are more accurate and robust than the standard EM approach
\end{abstract}

Keywords - component; Data fusion; Segmentation; Estimation and Maximization; MRI images

\section{INTRODUCTION}

In last decades, biomedical and medical image processing has become one of the most challenging fields of image processing and pattern recognition.

Brain segmentation consists of separating the different tissues: gray matter (GM), white matter (WM), cerebrospinal fluid (CSF) and probably abnormal (tumor) tissue.

The aim of segmentation of MRI Brain images is to: Study anatomical structure, Identify region of interest: locate tumor, others abnormalities, measure tissue size (to follow the evolution of tumor) and help in treatment planning prior to radiation therapy (radiation dose calculation).

However, the segmentation of MRI Brain images has remained a challenge in image segmentation. And this is due to partial volume effects, motion (patient movement, blood circulation and respiration), the existence of image noise, the presence of smoothly varying intensity in-homogeneity, the fact that different anatomical structures may share the same tissue contrast and large amounts of data to be processed. For these and others many approaches have been studied, including Methods based edge [1][2][3], methods based region [4][5], Methods based on thresholding [6][7], methods based artificial neural networks [8], data fusion methods [9], Markov random field methods [10] and hybrid Methods [11][12][13].
Segmentation process also helps to find region of interest in a particular image. The main goal is to make image more simple and meaningful. Estimation and Maximization approach (EM) is a unsupervised classification algorithm. EM algorithm has been developed by Dempster et al. in 1977 [14] and it has been used in several fields, in particular in images segmentation. The main idea of this approach is to approximate an image histogram by a set of Gaussian functions. So, the problem is how to estimate the parameters of the different Gaussian to approaching best the histogram. This estimation is done based on maximum likelihood. The EM approach provides probabilities of membership in different tissue class. Thus, EM can be used to model the partial volume effects, where a pixel may contain multiple tissue classes.

The data fusion, in imaging, is used mainly on radar images, satellite images, and aerial images. Recently, it is also applied in medical image. The increasing diversity of the medical image acquisition techniques motivated recent years much research aimed at developing models increasingly effective data fusion. Indeed, medical imaging, it may happen that no images available alone does not contain sufficient information. On the other hand the medical community entrusts each image type to an expert who has a partial diagnosis of the modality of his specialty and specialists exchange experiences and this confrontation comes the final diagnosis.

In this paper, the contribution is mainly to propose an architecture of a information fusion system guided by the prior knowledge and based on Estimation and Maximization approach to segment human MRI real Brain images.

The organisation of the paper is as follows. In section 2 the Estimation and Maximization approach of segmentation is reviewed and in section 3 describes briefly data fusion. Section 4 present a complete description of proposed segmenting approach using data fusion, where each step of the algorithm is developed in detail. Section 5 illustrates the obtained experimental results and discussions and section 6 concludes this paper.

\section{ESTIMATION AND MAXIMIZATION TECHNIQUE}

Estimation and Maximisation approach is one is a classical and unsupervised method, it has been proposed by Dempster in 1977 [14]. The idea of EM approach is inspired from a well 
know approach frequently used in statistic to describing distributions which is approximation using a combination. In this case, a no know distribution is estimate per a sum of parametric distributions. So the problem become to determinate the parameters of each distribution and its weight poids. In image segmentation, the EM approach is used to approximate an image histogram by a set of Gaussian distributions. So, the problem is how to estimate the parameters of the different Gaussian to approaching best the shape of histogram. Maximum likelihood is used for this estimation.

For each pixel, its intensity is xi. The intensity distribution of each tissue class is approximated by a Gaussian Gk of mean $\mu \mathrm{k}$ and $\sigma \mathrm{k}$ covariance matrix. Let $\pi \mathrm{ki}$ the priori-probability membership to a class $\mathrm{k}$ for each pixel $\mathrm{i}$ and Rki posterioriprobabilities membership to a class $\mathrm{k}$ calculated for each pixel $\mathrm{i}$ which are the searched labels.

EM algorithm has three main phases: Initialization phase, Estimation phase and maximization phase which are described as follows: the $\pi \mathrm{ki}$

Initialization phase: initialize Rki to the value given by

Estimation phase: calculus of classes parameters $\mu \mathrm{k}, \sigma \mathrm{k}$ using the flowing expressions:

$$
\begin{array}{r}
\mu \mathrm{k}=\frac{\sum_{i=1}^{N} R k i x i}{\sum_{i=1}^{N} R k i} \\
\sigma \mathrm{k}=\frac{\sum_{\mathrm{i}=1}^{\mathrm{N}} R k i x i(\mathrm{xi}-\mu \mathrm{k})(\mathrm{xi}-\mu \mathrm{k})^{\mathrm{T}}}{\sum_{i=1}^{N} R k i}
\end{array}
$$

Maximization phase: update of posterior probabilities Rki as follows:

$$
\mathrm{Rki}=\frac{\pi \mathrm{kiGk}\left(\mathrm{x}_{\mathrm{i}}\right)}{\sum_{\mathrm{i}=1}^{\mathrm{k}} \pi \mathrm{kiGk}\left(\mathrm{x}_{\mathrm{i}}\right)}
$$

Repeat the two phases: estimation and maximization until convergence.

\section{DATA FUSION}

Information fusion is to combine information (often imperfect and heterogeneous) from multiple sources to obtain better complete global information, to improve decision making and make better act. The terms "information" (numeric or symbolic) and "sources" cover many possibilities. In the same way, the notion of improvement depends wholly on the application.

Information fusion has evolved considerably in recent years in various fields, especially in vision and robotics, information sources have increased (sensors, a priori information, generic knowledge ... etc.). In general, each source of information is imperfect, it is important to combine several to get a better understanding of the all of the system.

MRI is a powerful tool to improve clinical diagnosis because it can provide various information in the form of image intensities related to the anatomy through a variety of excitation sequences (for example: T1, T2, and PD).
The proposed fusion involves the aggregation MRI images from different acquisition techniques. Data to be combined are so homogeneous, and depending on the type of image acquisition will provide more or less pronounced contrast between tissues or between parenchyma and pathology. One of the main interests of the fusion will be to exploit in particular the complementarity between the different images. Many applications can benefit from this technique. These include:

The detection of tumour regions:

MRI provides easy assess tumour extension, especially when contrast media are used. With certain acquisition techniques, the specificity is also greater in some cases to distinguish between tumor and oedema. The whole point is going to reside in a combination of these techniques with a more anatomical acquisition (weighted $\mathrm{T} 1$ type) to measure the tumor extension.

\section{Quantification of brain tissue volumes}

Because of its anatomical accuracy and variety of acquisition techniques, MRI is used to assess the distribution of different brain tissues following several contrasts. The volume quantification of these tissues is clinically fundamental to the study of many pathologies that affect the white matter, gray matter or cerebrospinal fluid, or simply for the measurement of volumes in healthy subjects. Information fusion can be doing at three conceptual levels corresponding to three types of information:

- Data fusion: it is essentially to marry low-level information such as primitives, in order to make information less noisy than that obtained with a single source of information.

- Decision fusion: it performs the combination of sophisticated information (numeric or symbolic) that can be considered as proposals for a decision.

- Models fusion: in this case, different approaches are set apart to fill imperfections affecting each of them independently.

A general information fusion problem can be stated in the following terms : given L sources S1, S2,...SL representing heterogeneous data on the observed phenomenon, take a decision di on an element $\mathrm{x}$, where $\mathrm{x}$ is higher level object extracted from information, and Di belongs to a decision space $\mathrm{D}=\{\mathrm{d} 1, \mathrm{~d} 2, \mathrm{~d} 3, \ldots, \mathrm{dn}\}$ (or set of hypotheses). In numerical fusion methods, the information relating $\mathrm{x}$ to each possible decision di according to each source $\mathrm{Sj}$ is represented as a number Mij having different properties and different meanings depending on the mathematical fusion framework. In the centralized scheme, the measures related to each possible decision $\mathrm{i}$ and provided by all sources are combined in a global evaluation of this decision, taking the form, for each $\mathrm{i}$ : $\mathrm{Mi}=$ $\mathrm{F}(\mathrm{Mi1}$, Mi2, Mi3, ..., Min), where $\mathrm{F}$ is a fusion operator. Then a decision is taken from the set of $\mathrm{Mi}, 1 \leq \mathrm{i} \leq \mathrm{n}$. in this scheme, no intermediate decision is taken and the final decision is issued at the end of the processing chain. In decentralized scheme decisions at intermediate steps are taken with partial information only, which usually require a difficult control or arbitration step to diminish contradictions and conflicts [15][16]. 
The three-steps fusion can be therefore described as:

Modeling of information: in a common theoretical frame to manage vague, ambiguous knowledge and information imperfection. In addition, in this step the Mij values are estimated according to the chosen mathematical framework.

Combination: the information is then aggregated with a fusion operator F. This operator must affirm redundancy and manage the complementarities and conflicts.

Decision: it is the ultimate step of the fusion, which makes it possible to pass from information provided by the sources to the choice of a decision di.[17]

I. Bloch [18] classified these operators in three classes defined as:

- Context independent and constant behaviour operators (CICB);

- Context independent and variable behaviour operators (CIVB);

- Context dependent operators (CD).

\section{METHODOLOGY}

Segmentation of brain images can separate different brain structures and detect possible pathologies, namely brain tumors. A good segmentation helps the doctor for making a final decision before his surgery. The main applications of the segmentation are morphometry, functional mapping and surface or volume visualization. Morphometry is the quantitative measurement of the positions, shapes and sizes of brain structures. It requires prior segmentation of these structures, and can identify, understand and follow the progression of diseases such as Alzheimer's or different tumors

The figure 1 shows the implementation of the proposed approach with its various stages:

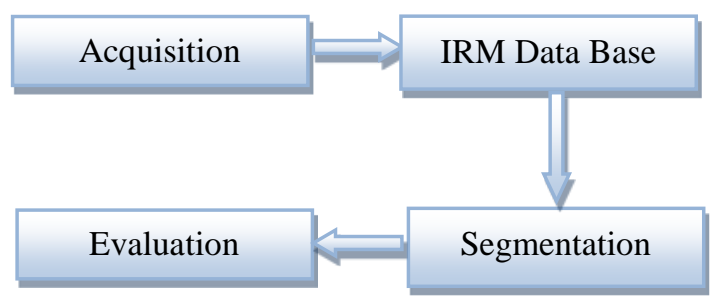

Fig. 1. Diagram of the various steps of the analysis system MRI images

\section{A. Acquisition:}

MRI Brain images are obtained by a Magnetic resonance imaging (MRI). Examination performed on a machine of high field $1.5 \mathrm{~T}$ according to the sequences:

- $\quad$ Axial and Sagittal Tl

- $\quad$ Axial T2 * Flair and diffusion.

- $\quad$ Coronal T2.

- Examination with and without gadolinium injection.

These MRI images are of different sections (axial, sagittal, and coronal) of healthy and pathological subjects. They are grouped into several sections.

\section{B. IRM DataBase:}

The format of images is the format DICOM (Digital Imaging and Communication in Medicine). This latter is a file used by most of the manufacturers of medical imaging; this standard was issued by the ACR (American College of Radiology) in association with the NEMA (National Electrical Manufacturers Association). The DICOM format is a file containing the image and patient data compressed (patient name, exam type, hospital, examination date, type of acquisition...etc.). To validate the proposed segmentation algorithms, a real database is used . These images are encoded in the DICOM format size 256x256 pixels. These images are grouped into several sections. Each image DICOM used has the following details:

- Format : 'DICOM'

- Color Type : 'grayscale’

- Modality : 'MR'

- Manufacturer: 'GE MEDICAL SYSTEMS'

- Institution Name: 'Medical Imaging Center of M'sila (DR S. F. Ghadbane)'

- Study Description: 'CEREBRAL'

- Series Description: 'FL:A/3-pl T2* FGRE S'

- $\quad$ Slice Thickness: 5

- Repetition time: 55.500

- Magnetic Field Strength: 15000

- Echo Time: 2.1000

- Spacing Between Slices: 10

- Spatial Resolution: 1.8750

- $\quad$ Flip Angle: 0

- Pixel pacing:[2x1 double]

The modality T1 is used to distinguish the different tissues such as: gray matter (GM), white matter (WM) and cerebrospinal fluid (CSF). However, The T2 modality do not allow to distinguish the GM from WM but highlight lesions and CSF.

\section{Segmentation}

Normally MRI Brain image can be classified in three classes: gray matter (GM), white matter (WM), cerebrospinal fluid (CSF). Each region has a certain gray level, for the T1 modality, the WM region has the gray level which tend to white one, the CSF region has the gray level which tend to black one and the grey level of GM region is between the both. The process of segmentation is done with EM using modalities fusion to separating the different tissues of MRI Brain images.

For MRI images fusion, context-based conjunctive operators are chosen because in the medical context, both images were supposed to be almost everywhere concordant, except near boundaries between tissues and in pathologic areas. 
In addition, the context-based behaviour allowed to taking into account these ambiguous but diagnosis-relevant areas. Then four operators of this class are retained, three of them are introduced in [18][19][20]:

$$
\begin{aligned}
& \text { OP1: } \pi_{T}(\mathrm{v})=\min \left(\pi_{T}^{T 1}(\mathrm{v}), \pi_{T}^{T 2}(\mathrm{v})\right)+1-\mathrm{h} \\
& \text { OP2: } \pi_{T}(\mathrm{v})=\max \left(\frac{\min \left(\pi_{T}^{T 1}(\mathrm{v}), \pi_{T}{ }^{T 2}(\mathrm{v})\right)}{h}, 1-\mathrm{h}\right) \\
& \text { OP3: } \pi_{T}(\mathrm{v})=\min \left(1, \frac{\min \left(\pi_{T}^{T 1}(\mathrm{v}), \pi_{T}^{T 2}(\mathrm{v})\right)}{h}+1-\mathrm{h}\right)
\end{aligned}
$$

with :

$$
\begin{aligned}
& \mathrm{h}=1-\sum_{v \in I m a g e}\left|\pi_{T}^{T 1}(\mathrm{v})-\pi_{T}^{T 2}(\mathrm{v})\right| / \mid \text { Image } \\
& \text { OP4: } \pi_{T}(\mathrm{v})=\frac{\left.\pi_{T}^{T 1}(\mathrm{v}), \pi_{T}^{T 2}(\mathrm{v})\right)}{2}
\end{aligned}
$$

The general algorithm for the EM using modalities fusion approach can be formulated as follows:

1) Set the parameters of the algorithm: C: number of cluster, $\varepsilon$ : convergence error.

2) For each image of section $j X j: j=1 \ldots S C$, which $S C$ is the section number.

3) Segmentation of each image section of each modality (T1, T2, PD) using EM provide posteriori-probabilities membership Rjt, $t=(T 1, T 2, P D)$, which $t$ is the modality.

4) Use one operator fusion $\mathrm{OPi}(t 1, t 2)$ then the output is membership matrix Rj,.

5) Assign all pixels to clusters by using the maximum membership value of every pixel.

\section{Evaluation :}

In addition to visual analysis, a quantitative evaluation is used on the above experimental results by different fusion algorithms. The selected quantitative criterions are standard deviation (SD), entropy (EN), spatial frequency (SF) and coefficient correlations (CC).

Standard deviation (SD): standard deviation is the square root of the variance, the variance of an image reflects the degree of dispersion among the grayscale values and the average value of gray levels. The larger the value is, the better fusion results are obtained.

$$
S D=\sqrt{\frac{\sum_{i=0}^{N-1} \sum_{j=0}^{M-1} F(i, j)}{M}}
$$

Entropy (EN): Entropy can effectively reflect the amount of information in certain image. The larger the value is, the better fusion results are obtained [21]:

$$
E N=\sum_{i=0}^{L-1} P_{f}(i) \log _{2} P_{f}(i)
$$

Where $\mathrm{P}_{u i}$ is the normalized histogram of the fused image to be evaluated, $\mathrm{L}$ is the maximum gray level for a pixel in the image.

Spatial frequency (SF): Spatial frequency can be used to measure the overall activity and clarity level of an image. Larger SF value denotes better fusion result [21]:

With

$$
S F=\sqrt{R F^{2}+C F^{2}}
$$

$$
\begin{aligned}
& R F=\sqrt{\left(\frac{1}{M(N-1)}\right) \sum_{i=0}^{M-1} \sum_{j=0}^{N-2}(F(i, j+1)-F(i, j))^{2}} \\
& \text { And } \\
& C F=\sqrt{\left(\frac{1}{N(M-1)}\right) \sum_{i=0}^{M-2} \sum_{j=0}^{N-1}(F(i+1, j)-F(i, j))^{2}}
\end{aligned}
$$

Coefficient correlation (CC): Coefficient correlation can show similarity in the small structures between the original and reconstructed images. Higher value of correlation means that more information is preserved [21]:

$$
C C=\frac{\sum_{j=1}^{N} \sum_{i=1}^{M}\left(x_{i, j}-\mu(A)\right)\left(x^{\prime}{ }_{i, j}-\mu(B)\right)}{\sqrt{\sum_{j=1}^{N} \sum_{i=1}^{M}\left(x_{i, j}-\mu(A)\right)^{2}\left(x^{\prime}{ }_{i, j}-\mu(B)\right)^{2}}}
$$

Where $\mu(\mathrm{A})$ and $\mu(\mathrm{B})$ are the mean value of the corresponding dataset.

\section{EXPERIMENTAL RESULTS}

The proposed approach Estimation and maximization using modalities fusion have been tested on real MRI brain images to certify their efficiency. It was acquired in medical imaging center of M'sila (DR S. F. Ghadbane). These MRI images are of different sections (axial, sagittal, and coronal) of healthy and pathological subjects of size $(256 \times 256)$. These images are grouped into 26 sections. The format of images is the format DICOM (Digital Imaging and size Communications in Medicine).

Examples of real images MRI present in this paper are extracted on axial sections of three modalities (T1, T2 and PD). The healthy images are segmented in four parts $(c=4)$ : background, white matter WM, gray matter GM and cerebrospinal fluid CSF. For tumoral subject, the images are segmented in five classes: background, WM, GM, CSF and the tumor. Standard deviation (SD), entropy (EN), spatial frequency (SF) and coefficient correlations (CC) are used to compare the performance of the adopted techniques for segmentation of MRI brain images.

To evaluate the performance of the proposed approach, two slices are presented for each adopted fusion operator and each fusion system. And obtained results are compared to those of EM. The two slices are: slice 16 from the healthy subject (Figure 2) and slice 22 from the tumor one (Figure 4) with three modalities (T1, T2 and PD). Figure 2, 3, 4 and 5 present for each example the segmented image using the EM and EM using system fusion ((T1, T2) (T1, PD), (T2, PD) and (T1, T2, $\mathrm{PD})$ ) with each operator(OP1, OP2, OP3 and OP4). Tables I, II, III, IV and V present for each example and for EM and EM using system fusion with each operator the detailed quantitative evaluation: (standard deviation (SD), entropy (EN), spatial frequency (SF) and coefficient correlations (CC)).

Table I and IV show that modality T2 provide the best segmentation. The use of modalities fusion has improved segmentation in terms of evaluation criteria (Table II, III and V). Segmentation by modalities fusion depends on modalities themselves and the used fusion operator: for example for the min operator (OP1) the best combination is T1 with T2. 


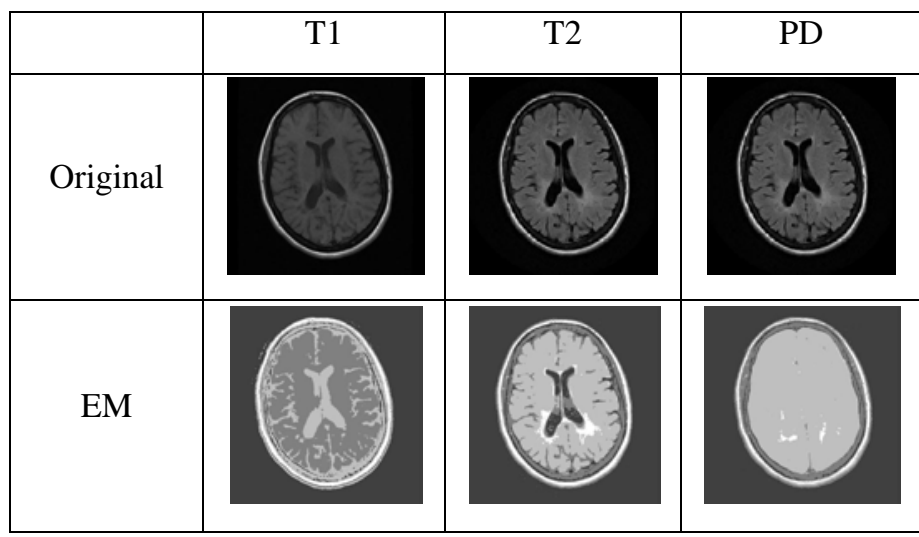

Fig. 2. Slice 16 from healthy MRI brain images and corresponding segmented image using EM algorithm for $\mathrm{c}=4$

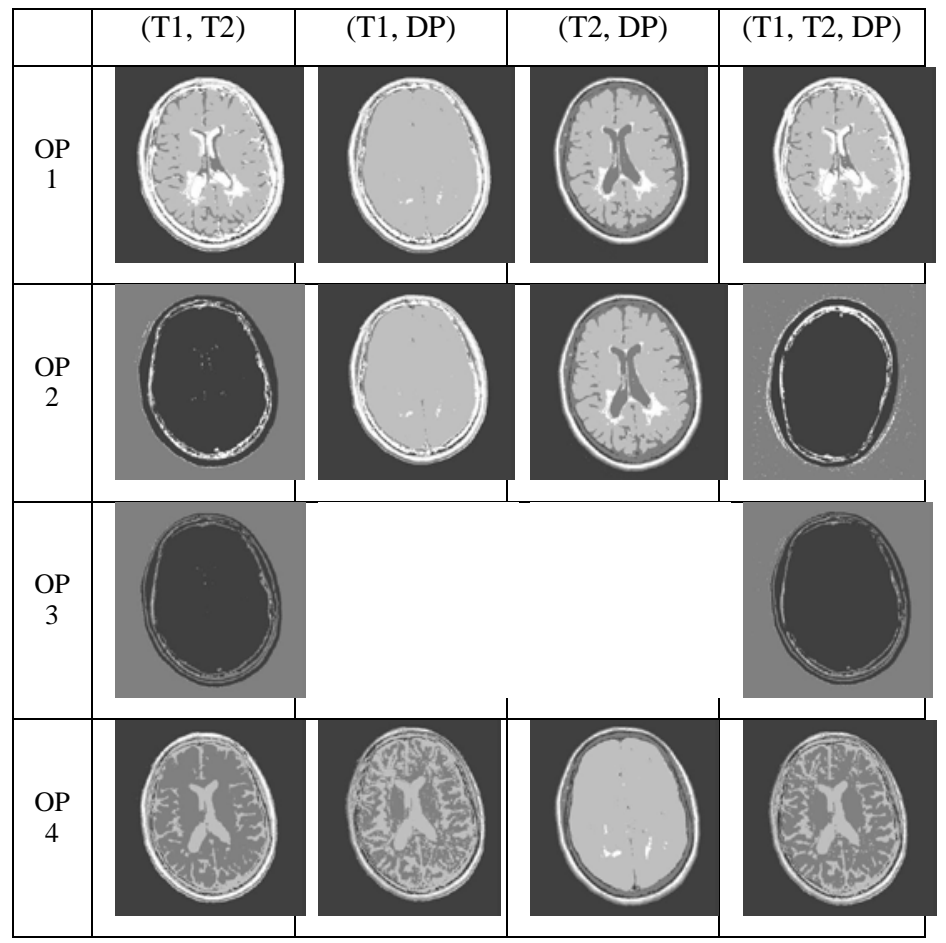

Fig. 3. The segmented results of the Slice 16 from healthy MRI brain images using the estimation and maximization algorithm using the four fusion operateur and the four fusion sytem ( $c=4)$

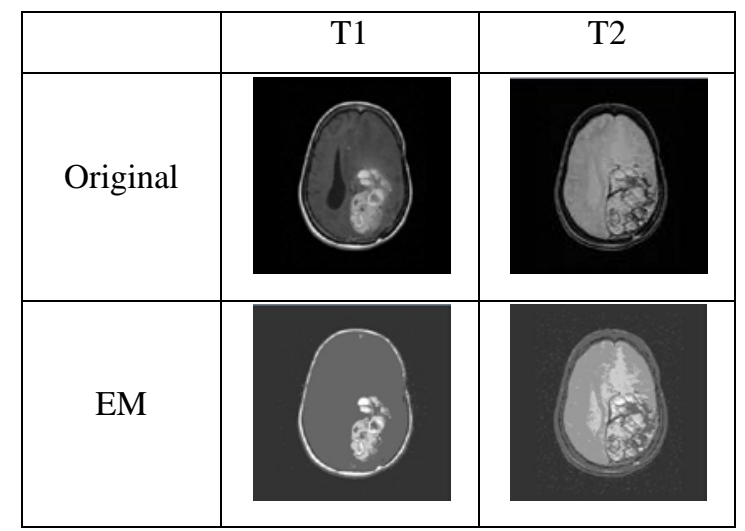

Fig. 4. Slice 22 from tumor MRI brain images and corresponding segmented image using EM algorithm for $\mathrm{c}=5$

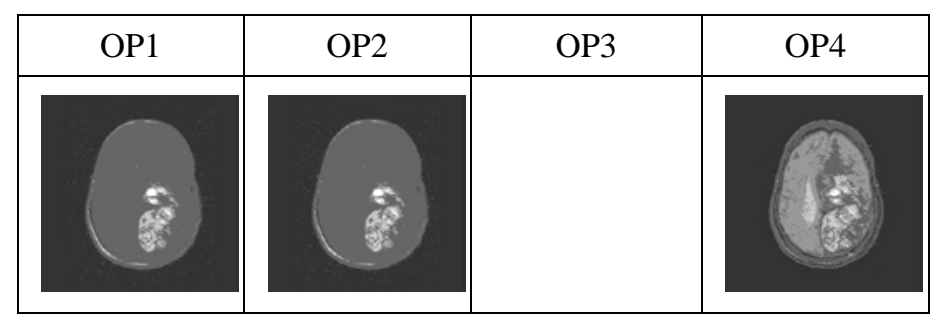

Fig. 5. The segmented results of the Slice 22 from tumor MRI brain images using the estimation and maximization algorithm using the four fusion operateur and the fusion sytem (T1, T2) with (c=5)

TABLE I. EXPERIMENTAL RESULTS USING EM FOR THE SLICE 16 FROM HEALTHY MRI BRAIN

\begin{tabular}{|l|l|l|l|l|}
\hline & CC & EN & SF & STD \\
\hline T1 & 0.957 & 1.468 & 26.661 & 10.085 \\
\hline T2 & 0.990 & 1.430 & 18.548 & 10.320 \\
\hline DP & 0.992 & 1.365 & 15.512 & 10.514 \\
\hline
\end{tabular}

TABLE II. EXPERIMENTAL RESULTS USING EM WITH THE FOUR FUSION OPERATOR AND THE FUSION SUSTEM (T1, T2) FOR THE SLICE 16 FROM HEALTHY MRI BRAIN

\begin{tabular}{|l|l|l|l|l|l|}
\hline & & CC & STD & SF & EN \\
\hline \multirow{2}{*}{ Op1 } & T1 & 0.932 & \multirow{2}{*}{10.953} & 35.400 & 1.537 \\
\cline { 2 - 3 } & $\mathrm{T} 2$ & 0.892 & & & \\
\hline \multirow{2}{*}{ Op2 } & $\mathrm{T} 1$ & -0.976 & \multirow{2}{*}{10.357} & 32.027 & 1.113 \\
\cline { 2 - 3 } & $\mathrm{T} 2$ & -0.925 & & & \\
\hline \multirow{2}{*}{ Op3 } & $\mathrm{T} 1$ & -0.840 & \multirow{2}{*}{10.251} & 16.567 & 1.004 \\
\cline { 2 - 3 } & $\mathrm{T} 2$ & -0.868 & & & \\
\hline \multirow{2}{*}{ Op4 } & $\mathrm{T} 1$ & 0.959 & \multirow{2}{*}{10.047} & 27.925 & 1.453 \\
\cline { 2 - 3 } & $\mathrm{T} 2$ & 0.848 & & & \\
\hline
\end{tabular}

TABLE III. EXPERIMENTAL RESULTS USING EM WITH THE FOUR FUSION OPERATOR AND THE FusION SUSTEM (T1, T2, PD) FOR THE SLICE 16 FROM HEALTHY MRI BRAIN

\begin{tabular}{|c|c|c|c|c|c|}
\hline & & CC & STD & SF & EN \\
\hline \multirow{3}{*}{ Op1 } & $\mathrm{T} 1$ & 0.923 & \multirow{3}{*}{10.975} & \multirow{3}{*}{34.846} & \multirow{3}{*}{1.546} \\
\hline & $\mathrm{T} 2$ & 0.891 & & & \\
\hline & DP & 0.942 & & & \\
\hline \multirow{3}{*}{ Op2 } & $\mathrm{T} 1$ & -0.964 & \multirow{3}{*}{10.430} & \multirow{3}{*}{34.468} & \multirow{3}{*}{1.182} \\
\hline & $\mathrm{T} 2$ & -0.918 & & & \\
\hline & DP & -0.965 & & & \\
\hline \multirow{3}{*}{ Op3 } & $\mathrm{T} 1$ & -0.830 & \multirow{3}{*}{10.235} & \multirow{3}{*}{17.5468} & \multirow{3}{*}{1.034} \\
\hline & $\mathrm{T} 2$ & -0.862 & & & \\
\hline & DP & -0.932 & & & \\
\hline \multirow{3}{*}{ Op4 } & $\mathrm{T} 1$ & 0.945 & \multirow{3}{*}{9.974} & \multirow{3}{*}{29.026} & \multirow{3}{*}{1.386} \\
\hline & $\mathrm{T} 2$ & 0.881 & & & \\
\hline & DP & 0.943 & & & \\
\hline
\end{tabular}

TABLE IV. EXPERIMENTAL RESULTS USING EM FOR THE SLICE 22 FROM TUMOR MRI BRAIN

\begin{tabular}{|l|l|l|l|l|}
\hline & CC & EN & SF & STD \\
\hline T1 & 0.991 & 1.237 & 13.808 & 8.619 \\
\hline T2 & 0.984 & 1.445 & 18.661 & 9.063 \\
\hline
\end{tabular}


TABLE V. EXPERIMENTAL RESULTS USING EM WITH THE FOUR FUSION OPERATOR AND THE FUSION SUSTEM (T1, T2) FOR THE SLICE 22 FROM TUMOR MRI BRAIN

\begin{tabular}{|c|c|c|c|c|c|}
\hline & & $\mathrm{CC}$ & STD & SF & EN \\
\hline \multirow{2}{*}{ Op1 } & $\mathrm{T} 1$ & 0.975 & \multirow{2}{*}{8.520} & \multirow{2}{*}{13.047} & \multirow{2}{*}{1.174} \\
\hline & $\mathrm{T} 2$ & 0.927 & & & \\
\hline \multirow{2}{*}{ Op2 } & $\mathrm{T} 1$ & 0.975 & \multirow{2}{*}{8.520} & \multirow{2}{*}{13.047} & \multirow{2}{*}{1.174} \\
\hline & $\mathrm{T} 2$ & 0.927 & & & \\
\hline \multirow{2}{*}{ Op3 } & $\mathrm{T} 1$ & 0 & \multirow{2}{*}{15.968} & \multirow{2}{*}{0} & \multirow{2}{*}{0} \\
\hline & $\mathrm{T} 2$ & 0 & & & \\
\hline \multirow{2}{*}{ Op4 } & $\mathrm{T} 1$ & 0.949 & \multirow{2}{*}{8.796} & \multirow{2}{*}{18.166} & \multirow{2}{*}{1.346} \\
\hline & $\mathrm{T} 2$ & 0.981 & & & \\
\hline
\end{tabular}

The fusion using the three modalities with the operator min (OP1) offer the best segmentation with a rate of correlation $\mathrm{CC}=0.923,0.891$ and 0.942 standard deviation $\mathrm{STD}=10.975$ spatial frequency $\mathrm{SF}=34.846$ and information entropy $\mathrm{EN}=$ 0.546 . The segmentation using the third operator provide white image for the tumour subject and for the fusion T1 with DP and T2 with DP. Fusion operator that has the best performance is the fourth one which confirms the qualitative improvement. As it can be seen, the adopted approach is very good and allows a good segmentation (Figure 3 and 5).

It can see that the main tissues (GM, WM and CSF) of the brain images are well separated for healthy subject and tumor region is well extracted for pathological subject. Fusion modalities and EM algorithm perform equally well in terms of the quality of image segmentation and leads to a good visual result.

\section{CONCLUSION}

Segmentation of medical images is still a vast field of research. The aim of this work is devoted to brain tissue segmentation from magnetic resonance images, in order to extract the tumor and also all other tissues (white matter, gray matter and CSF) by using of estimation and maximization with modalities fusion approach. This aggregation was performed by fusion operators that model doctor daily analysis confronted heterogeneous clinical data. The proposed approach Estimation and maximization using data fusion has been tested on real MRI brain images (healthy and pathological) to certify their efficiency. Experimental results show that: modalities fusion improves the segmentation of brain images. The fusion operators min and mean are the best for the segmentation of brain images and can deliver satisfactory performance to separating the different parts of an MRI brain real image. Further research is needed to improve the proposed approach. At level of modelling, other numeric or symbolic information can be integrated to increase the mass of available knowledge and at the combination one to design adaptive fusion operators for the combination of data in the medical field.

\section{ACKNOWLEDGMENT}

The authors would like to thank the medical imaging center of M'sila, Algeria, and specially Dr S. F. Ghadbane, for providing the MRI Brain images.

\section{REFERENCES}

[1] S. He, X. Shen, Y. Yang, R. He and W. Yan, "Research on MRI rain Segmentation Algorithm with the Application in Model -Based EEG/MEG”, IEEE Transactions on Magnetics, 37(5) 3741-3744. 2001.
[2] A. Yezzi, S.Kichenassamy, A. Kumar, P. Olver and A. Tannenbaum, “A geometric snake model for segmentation of medical imagery, Medical Imaging“, IEEE Transactions on, vol.16, no.2, pp.199-209, April 1997. Doi: 10.1109/42.563665.

[3] G. B. Aboutanos, J. Nikanne, N. Watkins and B. Dawant, "Model Creation and Deformation for the Automatic Segmentation of the Brain in MR Images“. IEEE Transactions on Biomedical Engineering, 46(11).1999.

[4] A. Kouhi, H. Seydarabi, A. Aghagolzadeh, "A Modified FCM Algorithm for MRI Brain Image Segmentation”. Machine Vision and Image Processing (MVIP), 2011 ( $7^{\text {th }}$ Iranian Digital Object Identifier: 10.1109/IranianMVIP.2011.6121551, pp 1-5. 2011

[5] I. Soesanti, A. Susanto, T.S. Widodo, M. Tjokronagoro. "optimized fuzzy logic application for mri brain images segmentation". International Journal of Computer Science \& Information Technology (IJCSIT) Vol 3, No 5, Oct 2011. pp 137-146. DOI : 10.5121/ijcsit.2011.3512.

[6] P. Kalavathi. "Brain Tissue Segmentation in MR Brain Images using Multiple Otsu's Thresholding Technique". The 8th International Conference on Computer Science \& Education (ICCSE 2013) April 2628, 2013. Colombo, Sri Lanka. 978-1-4673-4463-0/13/\$31.00 (C2013 IEEE 639

[7] C. Cheng; Y. Chen, T. Lin. "FCM Based Automatic Thresholding Algorithm to Segment the Brain MR Image" Machine Learning Machine Learning and Cybernetics, 2007 International Conference on Volume: 3 Digital Object Identifier: 10.1109/ICMLC.2007.4370358, pp: 1371 - 1376. 2007

[8] D.A Karras and B.G. Mertzios. "On Edge Detection in Mri Using the Wavelet Transform and Unsupervised Neural Networks”, EC-VIP-MC 2003. 4th EURASIP Conference focused on Video I Image Processing and Multimedia Communications, 2-5 July 2003, Zagreb, Croatia

[9] L. Gui, R. Lisowski, T. Faundez, P.S. Huppi, F. Lazeyras and M. Kocher. "Automatic Segmentation of Newborn Brain Mri Using Mathematical morphology”. 978-1-4244-4128-0/11/\$25.00 (C2011 IEEE.

[10] J. Tohka, I. D. Dinov, D.W. Shattuck, A.W. Toga. "Brain MRI tissue classification based on local Markov random fields" Magnetic Resonance Imaging, Volume 28, Issue 4, May 2010, pp 557-573

[11] N. Sharma, A. Ray, S. Sharma, K. Shukla, S. Pradhan and L. Aggarwal, "Segmentation and Classification of Medical Images using TexturePrimitive Features: Application of BAM-type Artificial Neural Network", Medical Physicists, vol. 33, pp. 119-126, 2008.

[12] M. Stella and B. Mackiewich, "Fully Automated Hybrid Segmentation of Brain, " Handbook of Medical Imaging: Processing and Analysis Management, I. Bankman, Ed, 2009.

[13] B.S. Anami, P.H. Unki. "A combined fuzzy and level sets based approach for brain MRI image segmentation” Computer Vision, Pattern Recognition, Image Processing and Graphics (NCVPRIPG), 2013 Fourth National Conference on Digital Object Identifier: 10.1109/NCVPRIPG.2013.6776216. pp 1 - 4. 2013.

[14] Dempster, A. P., Laird, N. M. and Rubin, D. B., Maximum likelihood from incomplete data via the EM algorithm. Journal of the Royal Statistical Society. 1977, Vol. 39, 1, pp. 1-38 .

[15] Bloch, I., and Maitre, H. 1997. Data fusion in 2D and 3D image processing: an overview. In proceedings of the $\mathrm{X}$ Brazilian symposium on computer graphics and image processing, Brazil, 127-134.

[16] Barra, V. and Boire, J. Y. 2001. A general framework for the fusion of anatomical and functional medical images. NeuroImage 13, 410-424.

[17] Lamiche C. Moussaoui A. Segmentation of MR Brain Images using a Data Fusion Approach International Journal of Computer Applications (0975 - 8887) Volume 36- No.12, December 2011

[18] Bloch, I. 1996. Information combination operators for data fusion : a comparative review with classification. IEEE Transactions in systems, Man. and Cybernitics 1, 52-67.

[19] Dubois, D. and Prade, H. 1992. Combination of information in the framework of possibility theory. In Data Fusion in Robotics and Machine Intelligence, M. AL Abidi et al. 
[20] Barra, V. 2000. Fusion d'Images 3D du Cerveau : Etude de Modèles et Applications. Thèse de doctorat, Université d'Auvergne.
[21] L. Yang, B.L. Guo, W. Ni, 2008, Multimodality medical image fusion based on multiscale geometric analysis of contourlet transform, Neurocomputing 72, P 203-211 